\title{
Evaluación de costo-efectividad de los nuevos anticoagulantes orales en pacientes con fibrilación auricular no valvular
}

\author{
Ángel Alberto García-Peña
}

Unidad de Cardiología, Hospital Universitario San Ignacio - Pontificia Universidad Javeriana, Bogotá, Colombia

Recibido el 17 de diciembre de 2015; aceptado el 7 de julio de 2016

Disponible en Internet el 20 de diciembre de 2016

\author{
PALABRAS CLAVE \\ Fibrilación auricular; \\ Ictus; \\ Tratamiento \\ anticoagulante; \\ Análisis de \\ costo-efectividad
}

\begin{abstract}
Resumen La fibrilación auricular es el trastorno más frecuente del ritmo cardíaco y una de las causas potencialmente tratables de cardioembolia y ataque cerebrovascular. La disponibilidad de nuevos anticoagulantes para pacientes con fibrilación auricular no valvular, plantea retos, derivados principalmente de los costos de su uso, perfiles de eficacia y seguridad, tolerancia y disponibilidad. Se llevó a cabo un análisis de costo-efectividad con base en un modelo de Markov, que permite comparar las estrategias de anticoagulación disponibles para fibrilación auricular no valvular en el país (apixabán, dabigatrán, rivaroxabán). La perspectiva empleada fue la del tercero pagador (sistema de salud colombiano), considerando solo costos médicos directos. Se siguieron las guías metodológicas para este tipo de estudios propuestas por ISPOR (International Society for Pharmacoeconomics and Outcomes Research) e INAHTA (International Network of Agencies for Health Technology Assessment). El horizonte temporal fue de 5 años, 10 años y toda la vida, en tanto que la tasa de descuento fue del $3 \%$. Luego de la evaluación del modelo, con base en los análisis de sensibilidad realizados y el umbral de costo-efectividad para Colombia, se encontró que para el caso base, las diferentes moléculas evaluadas, si bien son costo-efectivas, exceden el umbral propuesto para este trabajo, siendo rivaroxabán y dabigatrán las únicas estrategias costo-efectivas con un horizonte temporal a 10 años con una tasa de descuento del $3 \%$ y para un horizonte temporal de toda la vida y tasa de descuento del $3 \%$, las tres moléculas alcanzan el umbral de costo-efectividad establecido para Colombia. Dichas consideraciones son altamente sensibles al costo de los medicamentos.

(c) 2016 Sociedad Colombiana de Cardiología y Cirugía Cardiovascular. Publicado por Elsevier España, S.L.U. Este es un artículo Open Access bajo la licencia CC BY-NC-ND (http:// creativecommons.org/licenses/by-nc-nd/4.0/).
\end{abstract}




\section{KEYWORDS}

Atrial fibrillation;

Stroke;

Anticoagulation

therapy;

Cost-effectiveness

analysis

\section{Cost-effectiveness assessment of new oral anticoagulation drugs in patients with non-valvular atrial fibrillation}

\begin{abstract}
Atrial fibrillation is the most common heart rate disorder and one of the potentially treatable causes of cardioembolisms and cerebrovascular events. Availability of new anticoagulation drugs in patients with non-valvular atrial fibrillation poses challenges that mostly derive from usage costs, efficacy and security profiles, tolerance and availability. A cost-effectiveness analysis was conducted based on the Markov model, which allows to compare the available anticoagulation strategies for non-valvular atrial fibrillation in the country (apixaban, dabigatran, rivaroxaban). The third payer perspective (according to Colombian health system) was used, taking into consideration only the direct medical costs. The methodological guides followed were suggested by the International Society for Pharmacoeconomics and Outcomes Research (ISPOR) and the International Network of Agencies for Health Technology Assessment (INAHTA). The time frame was 5 years, 10 years and a lifetime, whilst the discount rate was $3 \%$. After assessing the model based on the conducted sensitivity analysis and the cost-effectiveness threshold for Colombia, it was revealed that for the baseline case the different molecules assessed, despite being cost effective, exceed the threshold proposed for this study. Rivaroxaban and dabigatran are the only cost-effective strategies in a 10 -year time frame with a discount rate of $3 \%$ and for a lifetime time frame and discount rate of $3 \%$ all three molecules reach the costeffectiveness threshold established for Colombia. These considerations are highly sensitive to the cost of the medications.

( $) 2016$ Sociedad Colombiana de Cardiología y Cirugía Cardiovascular. Published by Elsevier España, S.L.U. This is an open access article under the CC BY-NC-ND license (http:// creativecommons.org/licenses/by-nc-nd/4.0/).
\end{abstract}

\section{Introducción y marco teórico}

La fibrilación auricular es el trastorno más frecuente del ritmo cardíaco y una de las causas potencialmente tratables de cardioembolia y ataque cerebrovascular. Es un problema común en servicios de atención médica, que genera un aumento considerable de morbilidad, mortalidad y utilización de recursos del sistema de salud. Sin embargo, existen medidas para la prevención de ataques cerebrovasculares entre las cuales se cuenta la administración de antiagregantes plaquetarios y anticoagulantes, sustentada en guías de práctica clínica nacionales e internacionales ${ }^{1-5}$.

Dentro del abordaje integral de los pacientes con fibrilación auricular, se incluye la evaluación y estratificación del riesgo de ataque cerebrovascular, considerado como la principal complicación de esta enfermedad. Ese proceso de estratificación permite estimar el riesgo de sufrir ataque cerebrovascular en el siguiente año y elegir, en ausencia de contraindicaciones para su administración, una estrategia farmacológica que tiene como objetivo disminuir dicho riesgo, en cuyo caso la anticoagulación constituye la terapia estándar de tratamiento para la mayor parte de los pacientes, que según la evidencia científica disponible es segura y eficaz. Están disponibles varios medicamentos anticoagulantes que han demostrado beneficios significativos en la reducción de ataque cerebrovascular cardioembólico con bajo riesgo de sangrado, así que su uso rutinario es una práctica frecuente.

Entre los medicamentos disponibles para la anticoagulación oral en pacientes con fibrilación auricular no valvular está la warfarina, usada desde el siglo pasado y considerada como el estándar de tratamiento. Tiene interacciones farmacológicas con múltiples medicamentos y obliga a controles frecuentes de los niveles de INR (International Normalized Ratio) y de su dosificación, razón que ha llevado al desarrollo de nuevos anticoagulantes como apixabán, dabigatrán, edoxabán y rivaroxabán, ya disponibles en el mercado nacional (a excepción del edoxabán), que tienen como ventajas un perfil de seguridad más favorable, farmacocinética más previsible y menor número de interacciones farmacológicas en comparación con la warfarina. Sus principales limitaciones son la ausencia de antídotos aprobados y disponibles en el país para revertir su efecto anticoagulante, la contraindicación para su uso en pacientes con enfermedad renal crónica avanzada o bajo terapias de soporte renal, y su alto costo en Colombia. Esta última razón motivó el planeamiento de este trabajo, que busca, mediante una evaluación de costo-efectividad, definir los costos y razones de costo-efectividad incremental (RCEI), en el uso de estos medicamentos en el contexto del sistema de salud colombiano, a partir de información proveniente de los resultados de los experimentos clínicos que avalan en la literatura científica el empleo de estas moléculas, los costos propios del seguimiento, los insumos y el manejo de complicaciones.

\section{Metodología}

Se realizó un estudio de costo-efectividad (y costo-utilidad), con el objetivo de determinar la relación de costoefectividad (y costo-utilidad) de los nuevos anticoagulantes de administración oral (apixabán, dabigatrán y rivaroxabán) en comparación con warfarina, para la prevención de ataque cerebrovascular en pacientes con fibrilación auricular no valvular en Colombia. Se siguieron las guías 
metodológicas para este tipo de estudios propuestas por ISPOR (International Society for Pharmacoeconomics and Outcomes Research) e INAHTA (International Network of Agencies for Health Technology Assessment). Se programó el modelo en el programa TreeAge Pro versión 2014, en el cual se incluyeron las variables, costos y estados de salud, valores ajustados en pesos colombianos para el año 2014. La perspectiva empleada fue la del tercero pagador (sistema de salud colombiano), considerando solo costos médicos directos. El horizonte temporal fue de 5 años, 10 años y toda la vida y la tasa de descuento del $3 \%$.

\section{Tipo de modelo}

Se programó un modelo de Markov con ciclos de un mes (fig. 1). Para ingresar al modelo los pacientes debieron cumplir los siguientes criterios: diagnóstico de fibrilación auricular no valvular, con indicación de manejo con anticoagulación, candidatos para la administración de cualquiera de las tecnologías en evaluación o warfarina. El modelo tiene 13 estados de salud: fibrilación auricular no valvular, ataque cerebrovascular isquémico leve, moderado, severo, hemorrágico leve, hemorrágico moderado, hemorrágico severo, infarto agudo de miocardio (infarto agudo de miocardio), embolia sistémica, sangrado mayor, sangrado no mayor, muerte por otras causas y muerte. Las definiciones utilizadas se ajustaron a los estudios pivote de las moléculas en evaluación. Las probabilidades se estimaron a partir de la revisión de la literatura y de la información contenida en las guías de práctica clínica nacionales e internacionales. El modelo se evaluó mediante el método de análisis inverso. Dadas las consideraciones metodológicas del modelo de Markov, se consideraron los estados de salud descritos como finitos, exhaustivos y excluyentes; todos los estados son absorbentes a excepción del sangrado mayor y no mayor. Las unidades naturales utilizadas fueron: muerte, ataque cerebrovascular e infarto agudo de miocardio evitados, expresados según su RCEl (Razón de costo-efectividad incremental). Adicionalmente, se estimaron los años de vida ajustados por calidad (AVAC).

\section{Costos, insumos y probabilidades}

El valor monetario de los medicamentos fue consultado en el Sistema de Información de Precios de Medicamentos (SISMED), para el año 2014, complementando la información referente a precios y descripción de las presentaciones con el Sistema Vademécum Med-Informática-Catálogo Farmacéutico Nacional (Sistema VMI-CFN), creado por el Observatorio del Medicamento. Para la estimación de los costos de cada medicamento, y ante la ausencia de copias o genéricos de estas moléculas en el mercado nacional, se tomaron los precios reportados en las circulares emitidas por la Comisión Nacional de Precios de Medicamentos y Dispositivos Médicos (CNPMDM) como los valores máximos. Todos los costos están expresados en pesos colombianos. Para efectos del modelo, el uso de recursos corresponde al $100 \%$ debido a que cada medicamento se evalúa por separado. Esta información se presenta en las tablas 1 y 2. Para probabilidades derivadas de la efectividad y seguridad de los medicamentos, se extrajeron de metaanálisis, experimentos clínicos aleatorizados y experimentos clínicos aleatorizados controlados $^{6-11}$.

El proceso para la evaluación del consumo de recursos y el costo de los eventos en fase aguda y crónica (mantenimiento-rehabilitación) se realizó mediante la evaluación de información local, para lo que se tuvieron en cuenta las Guías de Práctica Clínica del Ministerio

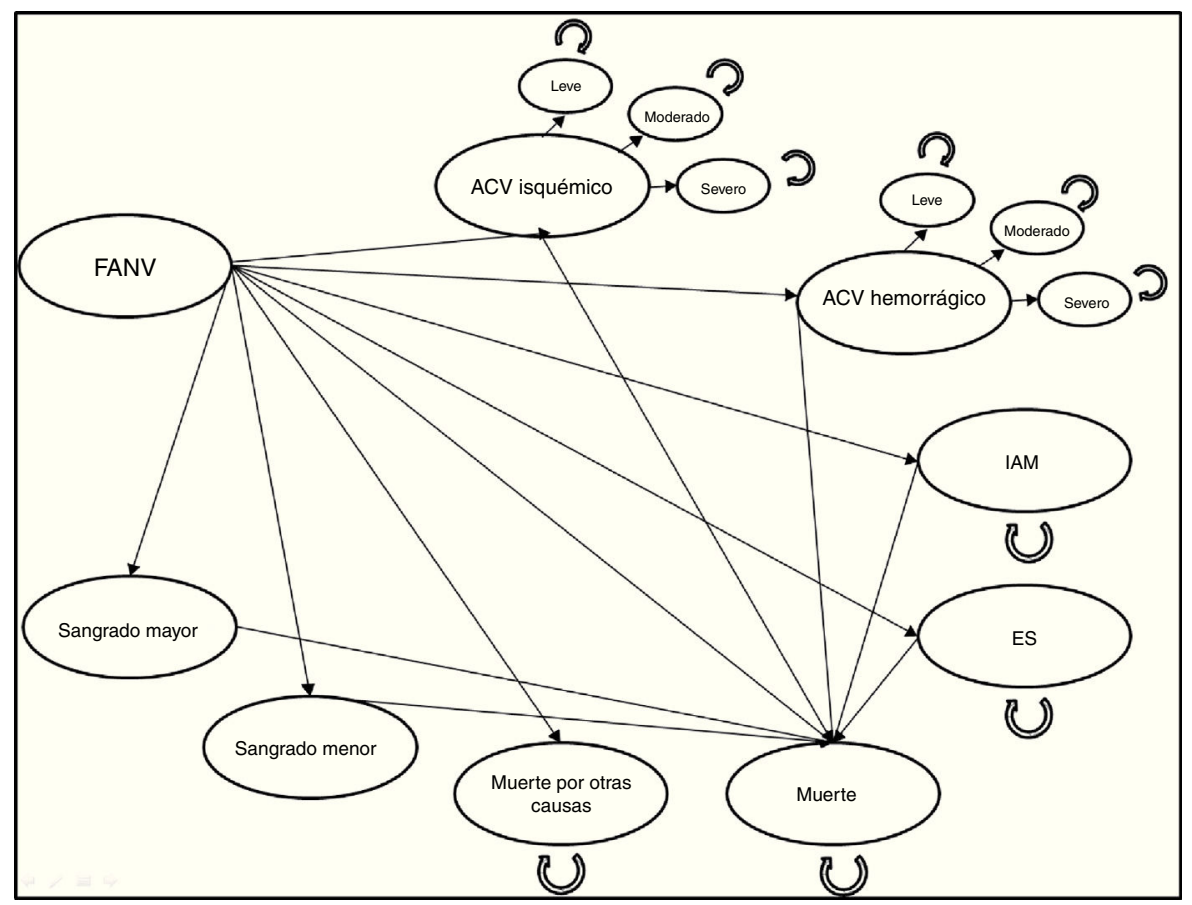

Figura 1 Modelo de Markov. 
Tabla 1 Costo unitario de anticoagulantes en fibrilación auricular no ventricular

\begin{tabular}{|c|c|c|c|c|c|}
\hline Medicamento & Unidad & Dosis diaria promedio $\mathrm{mg}$ & $\begin{array}{l}\text { Precio mínimo } \\
\text { por } \mathrm{mg}(\$)\end{array}$ & $\begin{array}{l}\text { Precio promedio } \\
\text { por } \mathrm{mg}(\$)\end{array}$ & $\begin{array}{l}\text { Precio máximo } \\
\text { por mg (\$) }\end{array}$ \\
\hline Warfarina & $\mathrm{mg}$ & $7,5 \mathrm{mg}(5-10 \mathrm{mg})^{*}$ & 7 & 69 & 175 \\
\hline Apixabán & $\mathrm{mg}$ & $10 \mathrm{mg}(5 \mathrm{mg}$ cada 12 horas) & 329 & 617 & 618 \\
\hline Dabigatrán & $\mathrm{mg}$ & $300 \mathrm{mg}$ ( $150 \mathrm{mg}$ cada 12 horas) & 7 & 19 & 21 \\
\hline Rivaroxabán & $\mathrm{mg}$ & $20 \mathrm{mg}$ & 237 & 239 & 256 \\
\hline
\end{tabular}

(\$) Precios expresados en pesos colombianos.

" Dado que existe variabilidad en la dosificación de la warfarina para alcanzar el objetivo terapéutico deseado, se considera que la dosis puede variar entre 5 y $10 \mathrm{mg} /$ día.

Tabla 2 Costo anual de los medicamentos anticoagulantes en fibrilación auricular no valvular

\begin{tabular}{lccc}
\hline Medicamento & Precio mínimo anual $(\$)$ & Precio promedio anual $(\$)$ & Precio máximo anual $(\$)$ \\
\hline Warfarina & $6.388 / 12.775^{*}$ & $62.963 / 125.925^{*}$ & $159.688 / 319.375^{*}$ \\
Apixabán & 1.202 .651 & 2.250 .464 & 2.254 .970 \\
Dabigatrán & 74.700 & 2.048 .941 & 2.273 .221 \\
Rivaroxabán & 1.732 .996 & 1.742 .201 & 1.872 .498 \\
\hline
\end{tabular}

(\$) Precios expresados en pesos colombianos.

* Dado que existe variabilidad en la dosificación de la warfarina para alcanzar el objetivo terapéutico deseado, se considera que la dosis puede variar entre 5 y $10 \mathrm{mg} /$ día.

de Salud y Protección Social para el manejo de Síndrome Coronario Agudo $^{12}$ y Dislipidemias ${ }^{13}$, en las cuales, mediante un proceso metodológico riguroso, se contó con la participación de expertos temáticos y metodológicos de talla nacional, quienes realizaron y validaron el consumo de recursos en los desenlaces de interés en cada una de las enfermedades presentadas en las guías, que presentan desenlaces comunes a los utilizados para el modelo económico del presente trabajo. Así mismo, se identificó información relevante sobre estos tópicos que fue utilizada en la construcción del modelo, aclarando que se ajustaron con la fórmula sugerida en la página web del MSPS http://mps1.minproteccionsocial.gov.co/evtmedica/ linea1.1/3.2\%20factor.html, en la cual, la técnica del descuento toma como referencia un año base y van actualizándose los flujos futuros de costos y resultados al año elegido como base, mediante la aplicación de la tasa de descuento. Así mismo, se hizo análisis de sensibilidad a través de la evaluación de la variación de los costos, según la inflación proyectada, sin encontrar variación en los resultados del análisis. Los valores relacionados en los diferentes documentos son relativamente similares. Para disminuir la incertidumbre derivada de eventos infrecuentes o cuya presentación clínica es variable, con grandes diferencias en el consumo de recursos para el manejo de la fase aguda y crónica, se procedió a modelar estos costos, con distribuciones específicas para cada variable.

\section{Información sobre utilidades}

Los pesos de las utilidades aplicados se extrajeron de la literatura, debido a que en Colombia no se cuenta con una base de datos sobre las preferencias de la población general respecto a los estados de salud evaluados. Dicha información se obtuvo de la Universidad de Tufts por medio del Center for the Evaluation of Value and Risk in Health (CEVR).
Posteriormente, se calculó la media aritmética simple de todos los estudios publicados según el estado de salud, para así estimar el peso promedio para el cálculo de los AVAC. Este resultado fue validado por un panel de expertos integrado por un cardiólogo, dos neurólogos, dos geriatras, un médico familiar, dos médicos generales y un paciente con ataque cerebrovascular.

\section{Modelamiento}

Para el proceso de modelamiento matemático se hicieron comparaciones indirectas mediante la metodología de metaanálisis en red (network metaanálisis), metodología que puede ser empleada cuando existen varias alternativas de tratamiento, mediante la comparación de las diferentes alternativas con un comparador común; para el caso de fibrilación auricular no valvular el comparador común es la warfarina. Para el proceso de cálculo se utilizó el método de Bucher para comparaciones indirectas a través de un comparador común en metaanálisis de ensayos con no más de dos brazos. Para el manejo de la variabilidad y la incertidumbre se efectuaron análisis de sensibilidad univariados y probabilísticos (con modelación de Montecarlo y curvas de aceptabilidad), para cada uno de los desenlaces de interés, con fin de comprobar si la variabilidad y la incertidumbre de los parámetros pueden modificar las conclusiones del estudio.

\section{Resultados}

\section{Resultados del caso base}

El caso base tiene un horizonte temporal de cinco años y una tasa de descuento de $3 \%$ anual. 
Tabla 3 Resultado del caso base. Horizonte temporal a 5 años, tasa de descuento 3\%

\begin{tabular}{lllllr}
\hline Alternativas & Costo & $\begin{array}{l}\text { Costo } \\
\text { incremental }\end{array}$ & AVAC & $\begin{array}{l}\text { AVAC } \\
\text { incremental }\end{array}$ & $\begin{array}{l}\text { Razón de costo-efectividad } \\
\text { incremental }\end{array}$ \\
\hline Warfarina & $\$ 3.545 .390$ & & 3,5144 & & \\
Dabigatrán 150 mg & $\$ 9.903 .759$ & $\$ 6.358 .369$ & 3,5895 & 0.0751 & $\$ 84.636 .068$ \\
Rivaroxabán & $\$ 9.966 .594$ & $\$ 6.421 .204$ & 3,5973 & 0.0829 & $\$ 77.453 .716$ \\
Apixabán & $\$ 12.017 .179$ & $\$ 8.471 .789$ & 3,5791 & 0.0647 & $\$ 130.926 .062$ \\
\hline
\end{tabular}

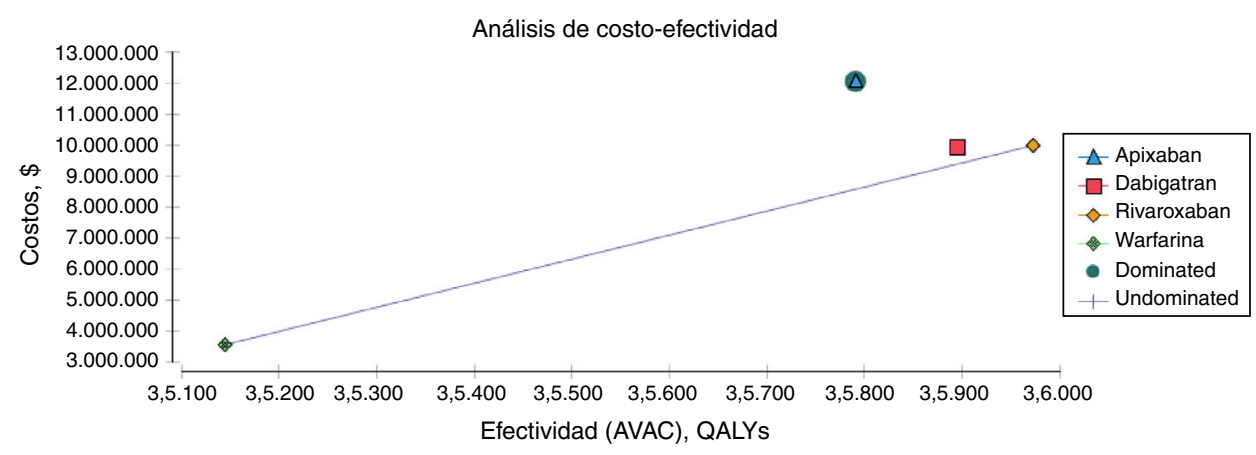

Figura 2 Resultado del caso base. Horizonte temporal a 5 años, tasa de descuento 3\%.

Tabla 4 Horizonte temporal a 10 años, tasa de descuento 3\%

\begin{tabular}{llllll}
\hline Alternativas & Costo & $\begin{array}{l}\text { Costo } \\
\text { incremental }\end{array}$ & AVAC & $\begin{array}{l}\text { AVAC } \\
\text { incremental }\end{array}$ & $\begin{array}{l}\text { Razón de costo-efectividad } \\
\text { incremental }\end{array}$ \\
\hline Warfarina & $\$ 6.923 .092$ & & 5,6624 & & \\
Dabigatrán 150 mg & $\$ 17.210 .980$ & $\$ 10.287 .888$ & 5,8830 & 0,2205 & $\$ 46.650 .165$ \\
Rivaroxabán & $\$ 17.450 .511$ & $\$ 10.527 .419$ & 5,9077 & 0,2453 & $\$ 42.923 .753$ \\
Apixabán & $\$ 20.816 .435$ & $\$ 13.893 .342$ & 5,8493 & 0,1869 & $\$ 74.340 .138$ \\
\hline
\end{tabular}

Los resultados se muestran en la tabla 3 y figura 2 . Adicionalmente, en la tabla 4 y figura 3 se aprecian los resultados para un horizonte temporal de 10 años y una tasa de descuento del $3 \%$ y en la tabla 5 y figura 4 para toda la vida.

\section{Interpretación resultados del caso base}

En el análisis de costo-efectividad, en el caso base con horizonte temporal de 5 años y tasa de descuento del $3 \%$, la opción más barata y menos efectiva es la warfarina, encontrando dentro de las alternativas en evaluación, que el apixabán logra 3,5791 AVAC, seguido por dabigatrán que logra 3,5895 y posteriormente rivaroxabán que logra 3,5973 AVAC, con altos costos para el sistema. Para el umbral de 10 años con tasa de descuento del 3\% el apixabán logra 5,8493 AVAC, seguido por el dabigatrán, que logra 5,883 AVAC, y luego el rivaroxabán con 5,9077 AVAC. Para el umbral de toda la vida con tasa de descuento del 3\% el apixabán logra 8,2458 AVAC, seguido por el dabigatrán que logra 8,3266

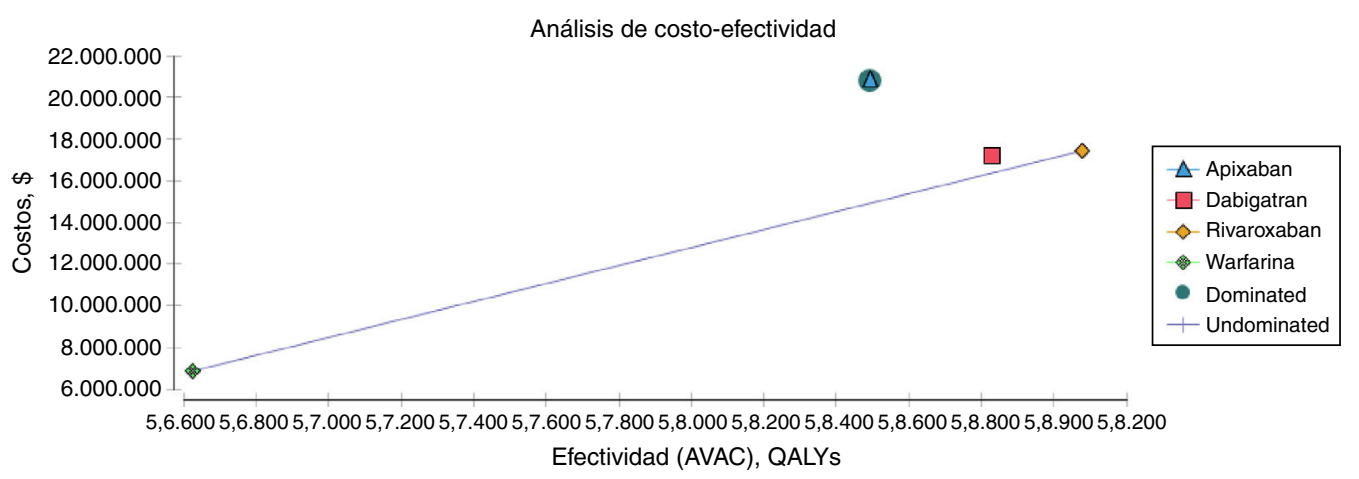

Figura 3 Horizonte temporal a 10 años, tasa de descuento 3\%. 
Tabla 5 Horizonte temporal toda la vida, tasa de descuento 3\%

\begin{tabular}{llllll}
\hline Alternativas & Costo & $\begin{array}{l}\text { Costo } \\
\text { incremental }\end{array}$ & AVAC & $\begin{array}{l}\text { AVAC } \\
\text { incremental }\end{array}$ & $\begin{array}{l}\text { Razón de costo-efectividad } \\
\text { incremental }\end{array}$ \\
\hline Warfarina & $\$ 11.901 .234$ & & 7,8262 & & \\
Dabigatrán 150 mg & $\$ 26.305 .143$ & $\$ 14.403 .909$ & 8,3266 & 0,5005 & $\$ 28.781 .873$ \\
Rivaroxabán & $\$ 26.943 .383$ & $\$ 15.042 .149$ & 8,3865 & 0,5603 & $\$ 26.846 .997$ \\
Apixabán & $\$ 31.681 .174$ & $\$ 19.779 .939$ & 8,2458 & 0,4196 & $\$ 47.145 .198$ \\
\hline
\end{tabular}

AVAC y posteriormente el rivaroxabán que logra 8, 3865 AVAC. Teniendo en cuenta que Colombia no cuenta con un umbral de costo-efectividad definido, se utilizó el umbral genérico propuesto por la Organización Mundial de la Salud (OMS), que corresponde a tres veces el producto interno bruto (PIB), per cápita, equivalente actualmente a \$47.594.859 para el año 2014 según reporte del Banco de la República. Así pues, para el umbral de 5 años ninguna de las tres moléculas en comparación con warfarina fue costo-efectiva, en el umbral de 10 años solo dabigatrán y rivaroxabán fueron costo-efectivos y con un horizonte temporal de toda la vida y una tasa de descuento del $3 \%$ las tres moléculas alcanzan el umbral de costo-efectividad para Colombia.

En el análisis de costo-efectividad, en el caso base con horizonte temporal de 5 años y tasa de descuento del 3\%, la opción más económica, aunque menos efectiva es la warfarina, situación que previsible considerando su bajo precio en Colombia y los hallazgos de eficacia con su uso en la prevención de los desenlaces de interés; sin embargo, los costos y AVAC logrados, no son despreciables cuando se comparan con las nuevas terapias, situación que se debe interpretar en el contexto de la sensibilidad que tienen los resultados al costo de los medicamentos. Dentro de las alternativas en evaluación, ninguna de las moléculas fue costo-efectiva con un horizonte temporal de 5 años, pero, a 10 años, el rivaroxabán y dabigatrán logran ser costo-efectivos, en tanto que para un horizonte temporal de toda la vida todas las moléculas alcanzan el umbral de costo-efectividad propuesto. Los análisis de sensibilidad realizados modificando la tasa de descuento $(0 \%, 5 \%, 7,5 \%$ y $12 \%)$, muestran como hallazgos relevantes que para un umbral de 10 años y una tasa de descuento mayor al $5 \%$ el dabigatrán deja de ser costoefectivo y para una tasa de descuento del $12 \%$ ninguna de las moléculas logra ser costo-efectiva. Para el umbral de toda la vida, con tasas de descuento mayores al 5\%, el apixabán deja de ser costo-efectivo, situación que no se presenta con dabigatrán y rivaroxabán ni modifica de forma sustancial las conclusiones en referencia con el caso base.

El análisis de sensibilidad determinístico se llevó a cabo permitiendo los valores mínimos y máximos de las probabilidades y costos con el fin de determinar qué parámetros generaban la mayor variabilidad respecto a los beneficios monetarios netos y podrían llegar a cambiar las conclusiones del estudio. El resultado del uso de los valores extremos en cada parámetro mostró, en el diagrama de tornado para costos, que las variables de mayor peso en el modelo son los costos derivados de los medicamentos, y que las demás variables clínicas tienen un impacto mínimo en los costos. Los costos de los medicamentos sí tienen gran influencia en los resultados; para ser costo-ahorradores respecto a la warfarina, los medicamentos tendrían que reducir su costo mensual para apixabán de 187.539 a 73.510 (un 61\%), dabigatrán de 145.215 a 82.698 (un 43\%) y para rivaroxabán de 145.183 a 88.429 (un 40\%). En el diagrama de dispersión de costo-efectividad de los medicamentos, se observa importante dispersión para las diferentes moléculas, como era de esperarse por su alta sensibilidad en los costos y pequeña diferencia en las efectividades con una ligera tendencia del rivaroxabán a ser el más costo-efectivo. En el análisis de sensibilidad, mediante el diagrama de tornados y valoración de la RCEI, entre los medicamentos (apixabán vs. dabigatrán, apixabán vs rivaroxabán y dabigatrán vs. rivaroxabán), se observa que el costo de los medicamentos vuelve a ser la variable de mayor impacto y en una menor proporción el infarto agudo de miocardio agudo.

El análisis de sensibilidad probabilístico se efectuó para permitir la variación en las probabilidades, las efectividades y los costos asociados a cada alternativa, y así mejorar la calidad de la información sobre la costo-efectividad de las terapias en evaluación. El análisis utilizó el método de simulación de Montecarlo de segundo orden, en el cual

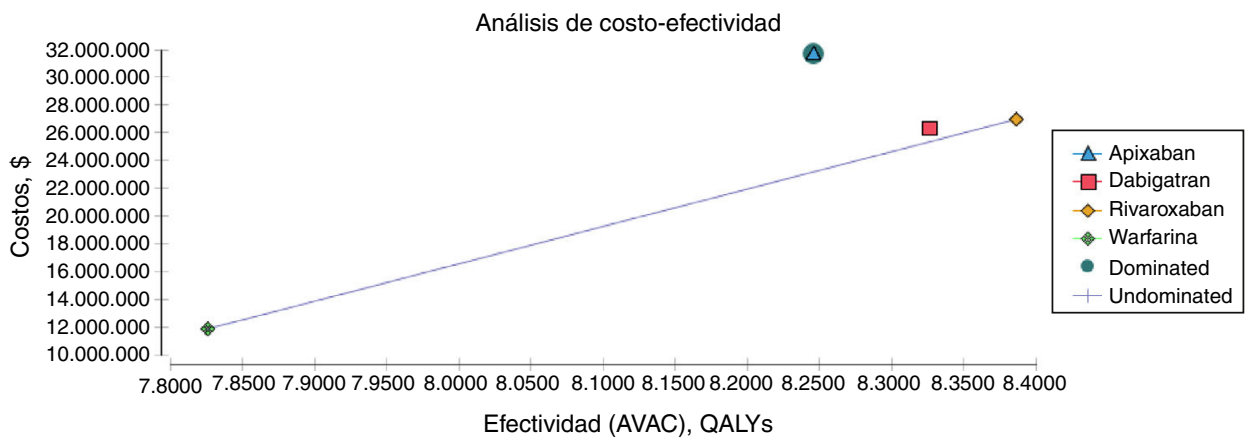

Figura 4 Horizonte temporal toda la vida, tasa de descuento 3\%. 


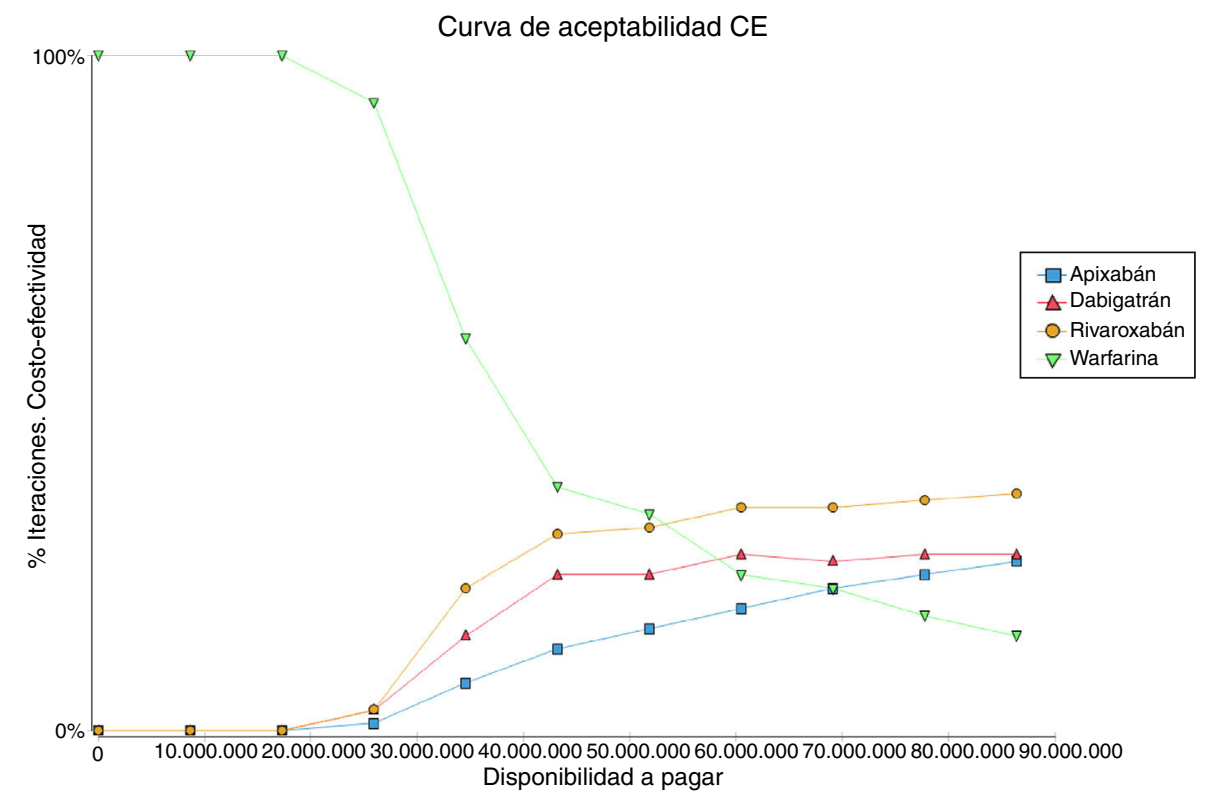

Figura 5 Curva de aceptabilidad.

se asignaron distribuciones para cada una de las variables incluidas. La simulación asignó valores aleatorios de forma simultánea a todas las variables incluidas en el modelo. En total se hicieron 1.000 simulaciones aplicando de manera aleatoria los valores de la distribución para cada variable. Estos resultados no muestran gran variación respecto a las estimaciones puntuales de costos y AVAC, y, por ende, no modifican las conclusiones generales. Así mismo, mediante una simulación de Montecarlo, se realizó un análisis sobre la frecuencia óptima de costo-efectividad, teniendo en cuenta el umbral propuesto previamente, y se determinó esta condición solamente en $12 \%$ de los casos para apixabán, $23 \%$ para dabigatrán, $29 \%$ para rivaroxabán y $36 \%$ para warfarina.

La curva de aceptabilidad muestra en el eje vertical el porcentaje de las simulaciones donde cada alternativa resultó costo-efectiva, y en el eje horizontal, la respectiva disponibilidad para pagar. Para una disposición a pagar superior a 40 millones por AVAC ganado, el rivaroxabán tiene mayor probabilidad de ser costo-efectivo, seguido por dabigatrán y posteriormente por apixabán (fig. 5).

\section{Discusión}

Un aspecto relevante en la toma de esta decisión y en especial, en el sistema de salud colombiano, son los costos derivados del uso de estos medicamentos, los cuales son relativamente altos en comparación con otros países con un perfil demográfico similar, planteando para el sistema un impacto presupuestal importante con la adopción de estas nuevas terapias, que no se encuentran incluidas en el Plan Obligatorio de Salud. Es claro, desde el punto de vista numérico, que estas terapias presentan beneficios en salud dados por la disminución de eventos cardiovasculares y mortalidad en comparación con warfarina, con perfiles de seguridad aceptables en relación con menor número de eventos de sangrado, situación que se traduce en un beneficio clínico neto a favor de los nuevos anticoagulantes orales, y que debe orientar a los tomadores de decisiones a generar políticas que permitan la adopción de estas terapias esperando este beneficio a un costo menor, mediante la regulación de precios, compra de medicamentos a escala nacional y eliminación de la intermediación económica entre las empresas farmacéuticas, sistema de salud y paciente, con miras a optimizar los recursos de salud, destinados para esta patología y sus complicaciones.

Respecto a los resultados, en especial al valor estimado por cada AVAC ganado y la RCEI, el factor determinante es el costo de los medicamentos, dado que el perfil de eficacia y seguridad es relativamente similar entre las moléculas, situación que está claramente identificada en la literatura. De ahí que el porcentaje de eventos no se modifica de forma significativa entre los nuevos anticoagulantes, y por tanto se considera que el modelo puede variar en sus resultados, si se modifica el costo de los medicamentos. Sin embargo, al evaluar la curva de aceptabilidad según la disposición a pagar por AVAC ganado, se resalta que la warfarina es la terapia dominante por debajo de los $\$ 40.000 .000$, situación que debe ser considerada en el contexto de la limitación de recursos para el sistema de salud, con beneficios que si bien son menores, no son despreciables, con la salvedad, que estos fueron logrados en poblaciones bajo seguimiento en estudios clínicos y un tiempo en rango terapéutico mayor al $55 \%$.

En el ámbito local existen trabajos similares, como el informe sobre el "Análisis de costo-efectividad de dabigatrán, rivaroxabán y apixabán comparado con warfarina para prevención de fenómenos tromboembólicos e isquémicos en pacientes adultos con fibrilación auricular no valvular en Colombia', presentado por el Instituto de Evaluación Tecnológica en Salud (IETS) en noviembre de $2015^{14}$, en el cual se hizo el modelamiento matemático con un modelo de Markov diferente al propuesto en este trabajo y un árbol de decisiones que simplifica los desenlaces relacionados con sangrado en el sistema nervioso central, modificaciones que cuentan con todo el soporte metodológico para su realización, y lo 
convierten en una propuesta interesante dado el abordaje del problema; sin embargo, sus conclusiones se encuentran en la misma dirección que las del presente trabajo.

Triana et al. ${ }^{15}$, presenta otro análisis de costo-efectividad en relación con una de las moléculas de interés para este trabajo (dabigatrán), con una metodología similar en la que se realiza modificación de los estados en el modelo de Markov (solo 6 estados), en un análisis a un horizonte temporal de 20 años, en el que se muestra la costo-efectividad de esta molécula para el sistema de salud colombiano. Sin embargo, en esta situación que tiene como limitación en su interpretación la incertidumbre relacionada con la efectividad a largo plazo y la expectativa de vida de la población colombiana al momento del inicio de la terapia, sí se mantienen los criterios de edad introducidos por el estudio pivote para dabigatrán (RELY).

Como limitaciones está la adopción de los supuestos para lograr el modelamiento matemático, que incluye el manejo de las definiciones de los desenlaces, la inclusión de comorbilidades de los pacientes, las cuales eran diferentes en las poblaciones de los estudios pivote al igual que su riesgo embólico según su estimación por $\mathrm{CHADS}_{2}$. No se incluyó en el modelo la variación en el tiempo de este puntaje debido al envejecimiento o presencia de eventos en su evolución, situación descrita en evaluaciones económicas similares, que si bien, es aceptable como ejercicio académico, no logra representar completamente todo el espectro clínico y evolutivo de esta población.

La significativa diferencia que existe en los perfiles demográficos y la exclusión de probabilidades de recurrencia y combinación de eventos, no fue evaluada por la gran incertidumbre de los datos, perfiles de riesgo y decisiones sobre la terapia anticoagulante posteriores a los mismos, que hacen parte de la vida real y son situaciones a las que se enfrentan los médicos a cargo de este grupo de pacientes, escenarios, en los que el juicio clínico, la individualización de la terapia y las prácticas propias de instituciones de salud, desempeñan un rol importante, que no fue evaluado en el presente modelo.

Es importante resaltar que las diferencias de riesgo en los distintos estudios pivote, fueron incluidas en el análisis bajo la metodología de comparaciones indirectas, si bien, existen diferentes metodologías en la forma de realizar comparaciones indirectas, como por ejemplo: ajustadas y no ajustadas, métodos bayesianos y simulación de eventos discretos las cuales presentan importantes limitaciones por los supuestos metodológicos necesarios para su realización. Todas estas comparaciones le confieren un alto grado de incertidumbre a los resultados en ausencia de comparaciones directas, información que por supuesto es deseable y quizás a futuro pueda, bajo un protocolo riguroso, dar la respuesta necesaria para disminuir la incertidumbre en la información sobre estos aspectos. Durante la evaluación de las revisiones sistemáticas, se hallaron puntos de vista importantes sobre este mismo aspecto, en los que se evalúan las diferencias en los resultados con las comparaciones indirectas y simulación de eventos discreta, encontrando que no existen diferencias relevantes en los resultados sobre evaluaciones económicas de estos medicamentos.

El uso de warfarina, principalmente los tópicos relacionados con el tiempo de rango terapéutico, frecuencia de monitorización, adherencia al tratamiento e interacciones farmacológicas, es extrapolado de datos de pacientes en estudios clínicos que tienen un seguimiento más estrecho y mayor conocimiento sobre su enfermedad, situación distante a la realidad del uso de este medicamento en la población general y en el contexto socio-cultural de Colombia.

\section{Conclusiones}

Luego de la evaluación del modelo, teniendo en cuenta los análisis de sensibilidad realizados y las limitaciones descritas previamente, se considera que los medicamentos evaluados tienen un mejor perfil de efectividad y seguridad en comparación con warfarina. Rivaroxabán y dabigatrán son las únicas estrategias costo-efectivas con un horizonte temporal a 10 años y tasa de descuento del 3\%, y para un horizonte temporal de toda la vida y tasa de descuento del 3\% las tres moléculas alcanzan a estar en el umbral de costo-efectividad establecido para Colombia.

\section{Responsabilidades éticas}

Protección de personas y animales. Los autores declaran que para esta investigación no se han realizado experimentos en seres humanos ni en animales.

Confidencialidad de los datos. Los autores declaran que en este artículo no aparecen datos de pacientes.

Derecho a la privacidad y consentimiento informado. Los autores declaran que en este artículo no aparecen datos de pacientes.

\section{Financiación}

El trabajo se financió mediante recursos propios del autor.

\section{Conflicto de intereses}

El autor declara no tener ningún conflicto de interés.

\section{Bibliografía}

1. National Collaborating Centre for Chronic Conditions. Atrial fibrillation: national clinical guideline for management in primary and secondary care. London: Royal College of Physicians; 2006.

2. Camm AJ, Kirchhof P, Lip GYH, Schotten U, Savelieva I, Ernst $S$, et al. Guidelines for the management of atrial fibrillation: the Task Force for the Management of Atrial Fibrillation of the European Society of Cardiology (ESC). Eur Heart J. 2010;31:2369-429.

3. Wann LS, Curtis AB, January CT, Ellenbogen KA, Lowe JE, Estes NAM, et al. 2011 ACCF/AHA/HRS focused update on the management of patients with atrial fibrillation (updating the 2006 guideline): a report of the American College of Cardiology Foundation/American Heart Association Task Force on Practice Guidelines. Circulation. 2011;123:104-23.

4. January CT, Wann LS, Alpert JS, Calkins H, Cleveland JC, Cigarroa JE, et al. 2014 AHA/ACC/HRS Guideline for the Management of Patients With Atrial Fibrillation: A Report of the 
American College of Cardiology/American Heart Association Task Force on Practice Guidelines and the Heart Rhythm Society. J Am Coll Cardiol. 2014;64:2246-80.

5. Guías de diagnóstico, tratamiento de la fibrilación auricular. Sociedad Colombiana de Cardiología 2007. Rev Colomb Cardiol. 2007;14 supl3:51-184.

6. Connolly SJ, Ezekowitz MD, Yusuf S, Eikelboom J, Oldgren J, Parekh A, et al. Dabigatran versus warfarin in patients with atrial fibrillation. N Engl J Med. 2009;361:1139-51.

7. Patel MR, Mahaffey KW, Garg J, Pan G, Singer DE, Hacke W, et al. Rivaroxaban versus warfarin in nonvalvular atrial fibrillation. New Eng J Med. 2011:883-91.

8. Lopes RD, Alexander JH, Al-Khatib SM, Ansell J, Diaz R, Easton JD, et al. Apixaban for reduction in stroke and other ThromboemboLic events in atrial fibrillation (ARISTOTLE) trial: design and rationale. Am Heart J. 2010;159:331-9.

9. Miller CS, Grandi SM, Shimony A, Filion KB, Eisenberg MJ. Meta-analysis of efficacy and safety of new oral anticoagulants (dabigatran, rivaroxaban, apixaban) versus warfarin in patients with atrial fibrillation. Am J Cardiol. 2012;110:453-60.

10. Baker WL, Phung OJ. Systematic review and adjusted indirect comparison meta-analysis of oral anticoagulants in atrial fibrillation. Circ Cardiovasc Qual Outcomes. 2012;5:711-9.
11. Gallagher AM, van Staa TP, Murray-Thomas T, Schoof N, Clemens A, Ackermann D, et al. Population-based cohort study of warfarin-treated patients with atrial fibrillation: incidence of cardiovascular and bleeding outcomes. BMJ Open. 2014;4:e003839.

12. Ministerio de Salud y Protección Social Colombia, Colciencias, Universidad de Antioquia. Guía de práctica clínica para el Síndrome Coronario Agudo. GPC-SCA. Bogotá; 2013.

13. Ministerio de Salud y de Protección Social. Guía de Práctica Clínica para la prevención, detección temprana, diagnóstico, tratamiento y seguimiento de dislipidemias en adultos; 2014.

14. Análisis de costo-efectividad de dabigatrán, rivaroxabán y apixabán comparado con warfarina para prevención de fenómenos tromboembólicos e isquémicos en pacientes adultos con fibrilación auricular no valvular en Colombia. Instituto de Evaluación Tecnológica en Salud (IETS). [Acceso Nov 2015]. Disponible en: http://www.iets.org.co/proyectos-en-curso/SiteAssets/ Documentacion\%20relacionada/Reporte\%20EE\%20dabigatran\% 20rivarixaban\%20y\%20apixaban\%20para\%20FA.pdf

15. Triana J, Castañeda C, Parada L, Otálora-Esteban M, Rosselli D. Costo-efectividad de dabigatrán comparado con warfarina para el tratamiento de pacientes con fibrilación auricular no valvular. Rev Colomb Cardiol. 2016;23:82-6. 